\title{
Gauging the impact of behavior change interventions: A tutorial on the Numbers Needed to Treat
}

\author{
Stefan L. K. Gruijters \& Gjalt-Jorn Y. Peters
}

This document is the full text of the article "Sauging the impact of behavior change interventions: A tutorial on the Numbers Needed to Treat" that has been resubmitted to (and is currently under review) at Psychological Methods.

This document can be cited as:

Gruijters, S. L., \& Peters, S. Y. (2019). Sauging the impact of behavior change interventions: A tutorial on the Numbers Needed to Treat. PsyArXiv. doi: 10.31234/osf.io/2bau7

The most current version of this manuscript is hosted at PsyArXiv at https://psyarxiv.com/2bau7 - please check that URL before citing this article.

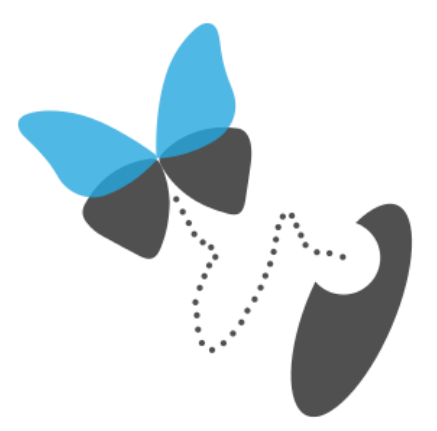


Running head: A tutorial on the Numbers Needed to Treat

Gauging the impact of behavior change interventions: A tutorial on the Numbers Needed to Treat.

\author{
Stefan L.K. Gruijters ${ }^{1}$ \\ Open University of the Netherlands \\ Gjalt-Jorn Y. Peters ${ }^{2,3}$ \\ Open University of the Netherlands \\ Maastricht University \\ *Author note*
}

${ }^{1}$ General Psychology, Faculty of Psychology \& Education Science, Open University of the Netherlands, Heerlen, the Netherlands

${ }^{2}$ Methodology \& Statistics, Faculty of Psychology \& Education Science, Open University of the Netherlands, Heerlen, the Netherlands

${ }^{3}$ Work and Social Psychology, Faculty of Psychology and Neuroscience, Maastricht University, Maastricht, the Netherlands.

Correspondence concerning this article should be addressed to Stefan Gruijters, Faculty of Psychology and Educational Science, Valkenburgerweg 177, 6419 AT, Heerlen, The Netherlands.

E-mail: mail@stefangruijters.nl

This work has been made available to the public in the form of a pre-print on PsyArxiv (https://osf.io/x4vqn). Parts of this work have been presented by Stefan L.K. Gruijters at the $31^{\text {st }}$ European Health Psychology Society conference, 2017, Padova, Italy.

Acknowledgements: The authors are thankful to N. Zonnebeld for sharing his insights into medical statistics. We are also thankful to J. DeCoster, P. Verduyn, and two anonymous reviewers for comments on an earlier version of this manuscript. 


\begin{abstract}
Effect size indices are valuable to research in applied psychology, but traditional measures (e.g. Cohen's $d$ or Pearson's $r$ ) are limited by their ability to convey practical information about intervention effectiveness. Researchers rely on concepts such as 'standardized mean difference' or 'explained variance' to express effectiveness. Practitioners, policymakers, and lay-people prefer concepts such as frequencies. Partial solutions have been offered by rules-of-thumb (e.g. Cohen's categories of 'small', 'moderate' and 'large'), but such pigeon-holing is somewhat arbitrary and of little nuance. We review, and provide a tutorial on, the Numbers Needed for Treat (NNT) statistic — an effect size index that originated in the medical literature. NNT fills the communicative gap between research and practice, and is particularly suited to gauge the impact of a behavior change intervention on a population level. NNT is defined as the number of people who need to be exposed to an intervention to achieve the desired change in one more individual, relative to a control condition. NNT has informational advantages: 1) it communicates effect magnitude in a frequency-format (number of people) making the impact of an intervention on a population transparent, and 2) it considers the population behavior base-rate to estimate this number. We adapt and extend the NNT index to suit applied psychology endeavors, and argue that the measure can strengthen the translation of intervention research to practice. The statistical procedure to estimate the NNT is explained, illustrated with concrete examples, and supplemented by script and functions to calculate the index in the $R$ - environment.
\end{abstract}

Keywords: Numbers Needed to Treat; effect size; applied research; intervention effectiveness; practical significance 


\section{Introduction}

Effect size (ES) indices are important products of science, but the value of a given ES index depends on the goal of the research project. One can distinguish (at least) two general aims of research. A large proportion of research in psychological science fits in the category of 'basic' — or 'fundamental' — research, where tests of theory take pole position. Other research lines rather seek applicable solutions to tangible problems—applied research projects, for example those interested in gauging the impact of an intervention on a population. It has been noted that the interpretation of traditional ES indices align rather poorly with the goals of applied research (e.g., Baguley, 2009, 2004). Traditional defaults include ES indices such as the standardized mean difference (e.g., Cohen's $d$ or $g$ ), various correlation coefficients (e.g., Pearson's $r$, Spearman's rho, or Cramer's V), measures that estimate explained variance proportions (e.g., $r^{2}, \eta^{2}, \omega^{2}$ ), and so forth. Researchers with an interest in gauging the impact of intervention tend to resort to such defaults, but these often do not convey the real-world implications of an effect (see also Kirk, 1996; Rosenthal, 1990).

For the aims of basic science, abstract quantifications or qualifications of findings are arguably necessary and perhaps sufficient to report. This is because basic science projects are primarily concerned with getting a sense of the truth of an hypothesis (e.g., Niiniluoto, 1993). Towards the end of supporting a truth-seeking, cumulative science, traditional ES estimation comes with various important and clear advantages over null-hypothesis significance testing (e.g., Cohen, 1994; Cumming, 2014; Gardner \& Altman, 1986; Greenland et al., 2016; Gruijters, 2016; Kirk, 1996; Lakens, 2013; Peters \& Crutzen, 2017; Wilkinson et al., 1999). But, despite the many advantages offered by traditional measures of ES we think that, for those with an eye on applicability, these indices do not convey the required information. Applied researchers need to communicate findings in a way that conveys the practical implications - the 'real-world' impact of an effect. 
Traditional ES indices do not give much insight into the change expected to occur in the population exposed to intervention. Correctly and meaningfully interpreting traditional ES measures requires a grasp of statistical concepts, which policymakers and practitioners (and other stakeholders of a given intervention) usually do not require for their day-to-day jobs and therefore often lack. One of the problems is that the metric of such indices cannot readily be converted to metrics familiar to people untrained in statistics. The metrics are non-intuitive, because standardized measures of ES communicate information by relying on concepts (e.g. standard deviation and variance) not typically used in people's thinking about magnitude (see also Baguley, 2009; Brooks, Dalal, \& Nolan, 2014).

This incongruity has become sufficiently salient and urgent that the 2016 joint conference of the European Health Psychology Society and the Division of Health Psychology of the British Psychological Association was themed "Behavior Change: Making an Impact on Health and Health Services". In the dedicated roundtable "Enhancing the Impact of Health Psychology on Policy and Practice", one of the conclusions was that the abstract level of scientific discourse does not lend itself well to communication with policymakers and practitioners. In similar vein, a council of representatives of the American Psychological Association (APA) noted that, to achieve effective translation of science into policy, researchers need to disseminate their research findings in accessible language and formats (APA, 2015).

The use of such accessible language and formats to express ES is, however, far from mainstream in papers written by applied psychologists. For example, the unit in which Cohen's $d$ expresses mean differences is 'standard deviations'. Does an estimated intervention effect of Cohen's $d=.90$ (falling in Cohen's 'large' range) imply that it is effective and worth implementing? The outcome that a behavioral intervention, compared to care as usual, has an ES of Cohen's $d=.90$ tells us little about the kind of change one could expect to observe in 
the real world, were the intervention to be implemented in a specific population. Traditional ES indices may aid researchers' in testing hypotheses, establish relative importance of variables, and make cross-study comparisons, but they limit our ability to communicate the real-world impact of interventions (see also Baguley, 2004, 2009).

In our view, then, reporting of traditional ES indices in applied research hardly qualifies as accessible dissemination of research findings. Therefore, the potential of applied psychology to impact policy and practice would be enhanced if the implications could be communicated less in the form of abstract statistical quantifications, and more using a measure that is tangible, practical, and intuitive. In this paper, we provide a tutorial on and introduce tools for estimating one such measure: the Numbers Needed to Treat (NNT).

The remainder of this paper is organized as follows: First, we provide a short review of previously proposed alternatives to traditional ES indices. Second, we proceed to discuss the definition, merits, and application of the NNT in applied research - initially in terms of its original use as an ES for binary outcomes. Third, we provide a step-by-step walk-through on how to estimate the NNT for continuous behavior outcomes in applied psychology. Finally, we will describe the function we implemented in package 'behaviorchange'(Peters, 2019) for the free and open source R software (R Development Core Team, 2017) ${ }^{1}$ that can be used to calculate the NNT based on Cohen's $d$ estimates. We will illustrate the procedures by working through a concrete example including several realistic scenarios. The examples are supplemented with an open source $R$ Markdown file available on the Open Science Framework (https://osf.io/x4vqn).

\section{Alternatives to traditional effect size measures}

We are certainly not the first to note the interpretational and communicative limitations of 'traditional' ES indices. Some authors (Baguley, 2009, 2004; Pek \& Flora, 2018) promote increased use of simple (unstandardized) rather than standardized ES. 'Simple' 
refers to the notion that an ES can also be expressed on its original scale, in raw units (e.g., raw mean difference or unstandardized regression coefficient), which is argued to provide various communicative and interpretational advantages over standardized indices (see also Wilkenson and the taskforce on statistical inference, 1999). Other attempts to forward suitable alternatives to traditional ES indices have instead taken a probabilistic turn to bolster interpretability. These approaches work from a shared premise that non-scientists, students, or simply 'the average Josephine' deal better with probabilistic information than traditional metrics. In this section, we provide a brief (and non-exhaustive) overview of some of these alternative metrics.

\section{Binomial effect size display}

Rosenthal \& Rubin (1982) introduced the 'Binomial effect size display' (BESD) which creates an easily interpretable two-by-two cross-table, by default using $r$ coefficients (see also Rosenthal, 1991, 2005, 1990). BESD dichotomizes a scaled outcome variable into categories of 'success' and 'failure' by assuming a 50\% base-rate occurrence. That is, if no intervention effect is present ( $r=0$ ), a probability of success (versus failure) of $P=.50$ is assumed in both the intervention and the control condition. The conditional probabilities consistent with the intervention effect are then computed. For example, given $r=.20$, a change from $40 \%$ 'success' in the control group to $60 \%$ in the experimental can be expected. Thus, the correlation coefficient estimates the success rate difference between an intervention and control group. The larger the correlation, then, the larger the success rate difference between a control and experimental condition.

The BESD highlights the interpretational weakness of traditional ES estimators. Even a correlation of $r=.10$, which according to traditional benchmarks would be branded 'small', corresponds to a $10 \%$ success rate increase in the BESD; such an effect can hardly be seen as irrelevant if this percentage expresses morbidity or mortality change in a large population (see 
Rosenthal, 1990). For squared correlation coefficients, the impression of irrelevance is even more readily evoked - that is, as Rosenthal (1990) pointed out, 'squaring the correlation coefficient tends to make it go away_vanish into nothingness' (p. 775). Thus, the apparent floor effect of ES magnitudes induced by some traditional indices may give an unjustifiable impression of effect irrelevance (see also Brooks et al., 2014).

\section{Probability of superiority}

Another class of probability-based ES indices expresses distribution differences by comparing individual scores across two groups. One parameter that can be estimated by comparing scores across two groups is the probability of superiority (PS; Grissom \& Kim, 2005, see also McGraw \& Wong, 1992; see also Ruscio, 2008; Ruscio \& Mullen, 2012). The PS conveys information about the relative position of two distributions by ranking the sample scores and calculating the probability that a case (selected at random) from the intervention group outscores one from the control group. That is,

$$
P S=P\left(Y^{1}>Y^{2}\right)
$$

If there is no effect of an intervention (Cohen's $d=0$; two distributions overlap completely), the PS equals 50\%. Such an estimate implies that a randomly picked individual from the experimental group has the same probability to outscore someone in the control group as an individual from the control group itself. The PS increases towards the asymptotes given large deviations from zero in terms of Cohen's $d$ values.

McGraw and Wong (1992) proposed the use of an estimator of PS they labeled the Common Language ES (CLES; see also Ruscio, 2008). This estimator is termed 'common language' because, according to these authors, no formal statistical knowledge to understand the metric of CLES. By making distributional assumptions (normality and variance homogeneity) the CLES is based on a z-score that estimates the probability of a difference 
score greater than zero (see McGraw \& Wong, 1992). Using group means and standard deviations, the CLES is estimated as follows:

$$
z(C L E S)=\frac{\bar{Y}^{1}-\bar{Y}^{2}}{\sqrt{S_{1}^{2}+S_{2}^{2}}}
$$

The CLES is then estimated by finding the probability that $z$ is smaller (the lower-tail probability) than the observed $z$ from a standard normal distribution. The CLES can be used as an estimator of the PS for continuous outcome variables, but the metric relies on parametric assumptions.

A more widely applicable nonparametric estimator of PS has been proposed as well the $A$ statistic (Vargha \& Delaney, 2000). A can be calculated for variables of ordinal and continuous measurement level and does not rely on distributional assumptions (see also Ruscio \& Gera, 2013). It can be computed as follows (see Vargha \& Delaney, 2002),

$$
A=P\left(Y^{1}>Y^{2}\right)+.5 P\left(Y^{1}=Y^{2}\right)
$$

The calculation in Equation 3 involves counting the number of times data points in one group are higher than in the other $\left(\mathrm{Y}^{1}>\mathrm{Y}^{2}\right)$, and $\mathrm{A}$ is subsequently corrected for ties $\left(\mathrm{Y}^{1}=\mathrm{Y}^{2}\right)$. These counts are converted to probabilities. The result is an estimate of the probability that an indvidual case in one group (e.g., experimental condition) outscores a case in another group (e.g., control group). $A$ is equivalent to a statistic found in signal detection theory (Swets, 1988), the area under the (receiver operating) curve (AUC) - which also provides an estimate of the PS (see Ruscio \& Mullen, 2012). Lastly, in addition to an estimator for the standardized mean difference, Cohen (1988) also described various probability-based effect size indices such as the U3 index. Simply put, this index expresses the probability that a given case in one condition (e.g., an experimental condition) scores above the mean value of the other group (e.g., a control condition). 


\section{Taking stock of probability-based ES measures}

It is important to note that several authors have criticized the use of BESD because its estimation of the success rate difference is applicable to only a limited suite of empirical findings (e.g., Hsu, 2004; McGraw, 1991). The CLES provides an easy to compute estimate of the PS (see Equation 2), and a major advantage is that raw data are not needed for its estimation so that it can be calculated with summary statistics. Conversely, the parametric assumptions it relies on make the measure susceptible to violations of those assumptions and to outliers - similar to Cohen's $d$ (Ruscio \& Mullen, 2012).

Vargha and Delaney's $A$ generalizes to more research designs and does not rely on distributional assumptions. For these reasons, $A$ has been advocated as the preferred estimator of PS (Hsu, 2004). One downside of the $A$ estimator is that researchers need access to raw data to compute the $A$ statistic. There are multiple options to compute the $A$ statistic using SPSS software - either based on a calculation of the Mann-Whitney $U$ statistic or the AUC dialogue. $A$ can be derrived from Mann-Whitney $U$ statistic by counting the number of times scores in one group outscore the other group (for details see Ruscio \& Mullen, 2012; Grissom \& Kim, 2002). Specifically,

$$
A=\frac{n_{a} n_{b}-U}{n_{a} n_{b}}
$$

$R$ software packages to calculate $A$ and other PS-estimators are also available and we refer readers to packages 'effsize' (Torchiano, 2017) or 'compute.es' (Del re, 2013).

Probability-based measures have one clear advantage compared to traditional ES indices: their metric ( $P$, or $P$ x 100) is relatively intuitive. Thus, for applied research purposes, such indices come a long way in communicating findings in an accessible format. Empirical research supports this claim. In two studies, Brooks et al. (2014) found support for the claim that probability-based ES measures - specifically, the BESD and CLES - are more 
understandable than traditional ES measures. Participants also perceived a training program as more effective, and were willing to pay more for the program, when the effect was communicated using BESD and CLES indices rather than $r$ and $r^{2}$. A recent study (Hanel et al., 2018) also found empirical evidence suggesting that probability-based indices specifically, Cohen's U3 and the PS- were rated as more informative than Cohen's $d$. These findings strengthen the notion central in this paper: applied research may benefit from alternative, practically meaningful, indices of ES. Probability-based ES indices have various advantages over traditional ES, and have arguably been under-utilized in psychological science. However, we propose to go beyond probability-based ES indices to express the practical relevance of applied research - in particular when the goal is to gauge intervention impact on a population.

\section{From probability towards frequency}

The value of applied psychological research is strongly connected to its translation to practice and ultimately its ability to inform how to improve the well-being of people, groups, and societies. Thus, a measure that aligns with the aims of applied research ideally conveys information that can be readily translated to the real-world and is informative to lay people. In our view, this implies that a practical measure of ES requires (1) a metric which is optimally understandable and interpretable for a non-scientific audience, and (2) expresses information that directly relates implications of an intervention to the population under study. While methods such as the BESD, but most notably estimators of PS (CLES and A) clearly go a long way towards meeting criterion (1) relative to traditional metrics, they do leave room for improvement on point (2).

One important reason for this is that both indices rely on estimates of single-event probabilities. A vast literature on judgment and decision-making, sprung from the seminal work by Kahneman and Tversky (Kahneman, Slovic, \& Tversky, 1974; Kahneman \& 
Tversky, 1972; Tversky \& Kahneman, 1983), illustrates that people experience difficulties when dealing with probability information. Moreover, studies (Cosmides \& Tooby, 1996; Gigerenzer, 1996; Gigerenzer, Hoffrage, Mellers, \& McGraw, 1995; Sloman, Over, Slovak, \& Stibel, 2003) have suggested that numerical information-processing may improve when people deal with frequency formats (e.g., 1 out of 10) rather than single-event probabilities (e.g, .10).

A second reason is that single-event probabilities give limited information about change on a population level -what change will a behavioral intervention enable in the population we expose to it? This issue is of particular relevance to applied researchers testing interventions in a well-defined target population. For example, health care institutes may wish to implement an intervention to increase vaccination uptake among medical personnel (Lehmann, Chapman, Franssen, Kok, \& Ruiter, 2016), and these institutes need to be able to make decisions whether such an intervention is worthwhile - often without any formal statistical knowledge. Organizations may wish to encourage employees to participate in training; both to promote their employees' sustainable employability and to maintain their own competitive advantage - and want to know how effectively a specific intervention encourage their employees to participate in these trainings (Fleuren, De grip, Kant, \& Zijlstra, 2018). For applied research, ES indices are required that connect research to practice.

We advocate using the Numbers Needed to Treat statistic (NNT; Cook \& Sackett, 1995; Laupacis, Sackett, \& Roberts, 1988) in the context of behavior change interventions. NNT can be defined, put simply, as the number of people who need to be exposed to an intervention to achieve the desired behavior change in one more individual relative to a control condition (formal definitions will be discussed in a later section). We suggest that to strengthen the link between research and practice, an understandable and practically meaningful ES needs to be estimated and reported. The NNT reviewed in this paper is one 
means towards this end. Unlike traditional measures of ES, the NNT index is practical and intuitive: it expresses the size of effect in a metric that conveys information about the 'realworld' implications of an intervention in the population (Citrome \& Ketter, 2013; see also Hilton, Reid, \& Paratz, 2006). The metric does not require a cognitive grasp of statistical concepts such as 'standard deviations', 'variance' or 'covariance', but communicates in an intuitive frequency-format which bolsters interpretability and understandability.

\section{Numbers Needed to Treat: A basic definition}

The Numbers Needed to Treat (NNT) index was developed in the context of medical statistics (Altman, 1998; Altman \& Andersen, 1999; Cook \& Sackett, 1995; Laupacis et al., 1988). Medical professionals had a growing need for intuitive effect measures, able to easily inform clinical practitioners about optimal treatment choices. The NNT provides a more intuitive index compared to Odds Ratio or Relative Risk because its primary metric is number of persons. This metric is less abstract, and to most clinicians and patients, more familiar, than magnitude of a standardized change on a medical outcome measure. In medical statistics, for treatments that aim to increase an event rate (ER), NNT is defined as:

$$
N N T=\frac{1}{E E R-C E R}
$$

Alternatively, treatments aiming to decrease a certain undesirable event rate can use the reverse operation to estimate NNT:

$$
N N T=\frac{1}{C E R-E E R}
$$

In Equations 5 and 6, EER is the experimental group event rate, and CER is the control group event rate. In both groups, an 'event' is a discrete outcome that a treatment aims to either promote or prevent, depending on what outcome is desirable. In medicine, examples of ERs 
are the number of patients that undergo a cardiac event, suffer a stroke, or recover from a traumatic injury. The NNT, then, compares the ER in the control group with the ER in the experimental group, expressing how many individuals would need to be given the treatment to have one more favorable event (e.g. recovery) relative to the CER. A small NNT is therefore indicative of a large treatment effect. For example, NNT=5 implies that out of every 5 individuals exposed to an intervention, one individual will show the desired change. Note, however, that NNT is relative to a reference condition (the CER), which slightly complicates its interpretation. Consider that for any given outcome (e.g., quitting smoking), there is always a proportion of the control group who will do this without intervention. More precisely, then, the NNT $=5$ estimate expresses that on average, out of every 5 individuals exposed to an intervention, one more person quits smoking over and above the number of people quitting without intervention (CER). Of course, the intervention could also be compared to a current or alternative treatment rather than a no-treatment CER.

The NNT measure is statistically related to other ES measures (see Figure 1 for basic computations) such as Area Under the (receiver operating characteristic) Curve (AUC), odds ratio (OR), relative risk ratio (RR), and success rate difference (SRD, also known as risk difference). These indices express how an ER in the experimental group differs from that in the control group, though with somewhat different ways of probabilistically expressing the CER and EER difference (Furukawa \& Leucht, 2011; Kraemer \& Kupfer, 2006). Methods to extract the NNT (but also SRD and RR) from a logistic regression model are described elsewhere (e.g., Austin, 2010). 


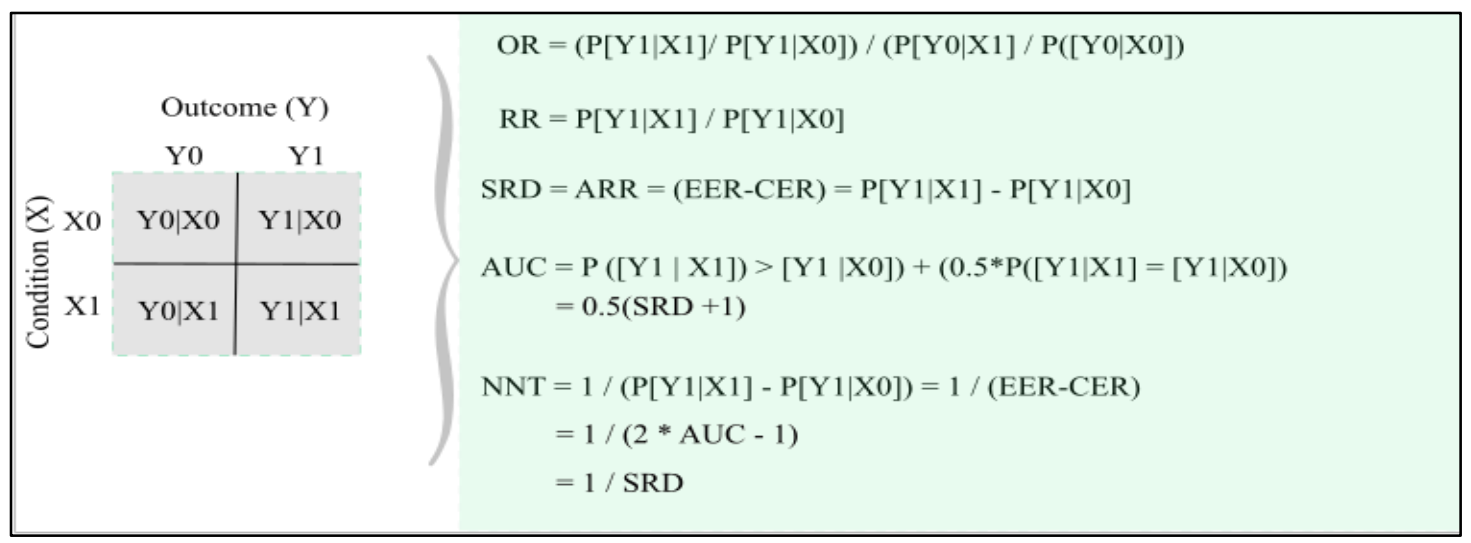

Figure 1. Basic binary effect size computations and relations.

SRD, AUC and NNT conversions are based on Kraemer \& Kupfer (2006). Abbreviations: OR $=$ Odds Ratio; RR = Relative Risk; SRD = Success Rate Difference; ARR= Absolute Risk Reduction; EER= Experimental Event Rate; $\mathrm{CER}=$ Control Event Rate; $\mathrm{AUC}=$ Area Under the $($ ROC) Curve; NNT = Numbers Needed to Treat.

In medicine, NNT contributes to communicating effectiveness of medical interventions to both physicians and patients. Consider the following example borrowed from Austin (2010). One medical intervention to prevent recurrence of acute myocardial infarction is to prescribe statins - a lipid-lowering medicine shown to decrease mortality due to cardiovascular disease. Using a large dataset of patients from Ontario (as cited in Austin, 2010), it is estimated that prescribing statins leads to an absolute reduction in 3-year mortality by $3.3 \%$. The NNT point-estimate was 30.3 . What this implies is that for every 31 or so people treated with statins (in a population with cardiovascular disease), statins may prevent 1 death over a 3-year period - relative to a population not receiving statins. Compared to its alternatives, the NNT's foremost strength is that it makes the effect of a treatment concrete in terms of numbers - it communicates intervention effects in a way that makes population impact of treatment tangible.

\section{NNT in psychological research}

In psychological intervention research, events can be defined in terms of specific behavior change objectives that programs aim to address, by attempting to either increase or 
decrease a specific behavior. Adequate intervention development and evaluation requires that the goals are formulated with sufficient specificity (Bartholomew Eldregde et al., 2016). For example, instead of aiming to "promote exercise", interventions will often aim to "promote engaging in physical activity for at least 30 minutes, at least five times a week". Such an intervention could define an ER as 'the proportion of people who engage in physical activity, at least for 30 minutes, five times a week'. In the context of sexual health, a typical ER could be described as 'the proportion of people who consistently use condoms when having sex outside a committed relationship', or 'the proportion of people who get screened for sexuallytransmitted infections after having unsafe sex'.

If researchers have estimates of the frequency of these discrete behaviors, in both the control and experimental populations, it is possible and straightforward to calculate the NNT given the above formulas. The formula would then return a discrete number indicating how many people need to be exposed to the intervention, to achieve favorable change in one more individual relative to the control condition. Unfortunately, however, discrete event rates are often not available for behavior change interventions. Binary behavior measures are not always feasible outcome measures - and, indeed, are statistically often not desirable (Cohen, 1983; DeCoster, Iselin, \& Gallucci, 2009; MacCallum, Zhang, Preacher, \& Rucker, 2002). Researchers therefore often use scaled outcomes, where magnitude of effect is commonly expressed in terms of standard deviations or variance proportions. In the following sections, we will extend the NNT procedure to provide for continuous data.

\section{Estimating NNT for scaled outcomes: the relevance of prevalence}

Authors (Furukawa, 1999; Furukawa \& Leucht, 2011; Hasselblad \& Hedges, 1995; Kraemer \& Kupfer, 2006; Suissa, 1991) have proposed different methods to estimate the NNT based on scaled data (for a comparison see, Da Costa et al., 2012; Thorlund et al., 2011). Equation 7 expresses Furukawa and Leucht's (FL) method of converting a Cohen's $d$ value 
into the NNT. The equation uses the cumulative standard normal probability distribution function to estimate the effect an intervention has (Cohen's $d$ ), above and beyond the ER observed in a control group. To do so, an estimate of the prevalence of events in the control group (CER) must be provided.

$$
N N T(F L)=\frac{1}{\Phi(d-\Psi(C E R))-C E R}
$$

In Equation 7, $\Psi$ represents the quantile function of the normal distribution, and $\Phi$ represents the distribution function of the normal distribution. To estimate the NNT, Equation 7 uses Cohen's $d$ to modify the CER, to estimate how many more desirable events we can expect if we would expose individuals to our intervention relative to a control condition (see Figure 2). Commonly, such a control condition is tantamount to doing nothing towards the promotion or prevention of specific behaviors (cf. 'the baseline'). But the CER could also describe the prevalence of behavior under a currently used behavior change method ( $c f$. 'care as usual'), to which a novel intervention is then compared.

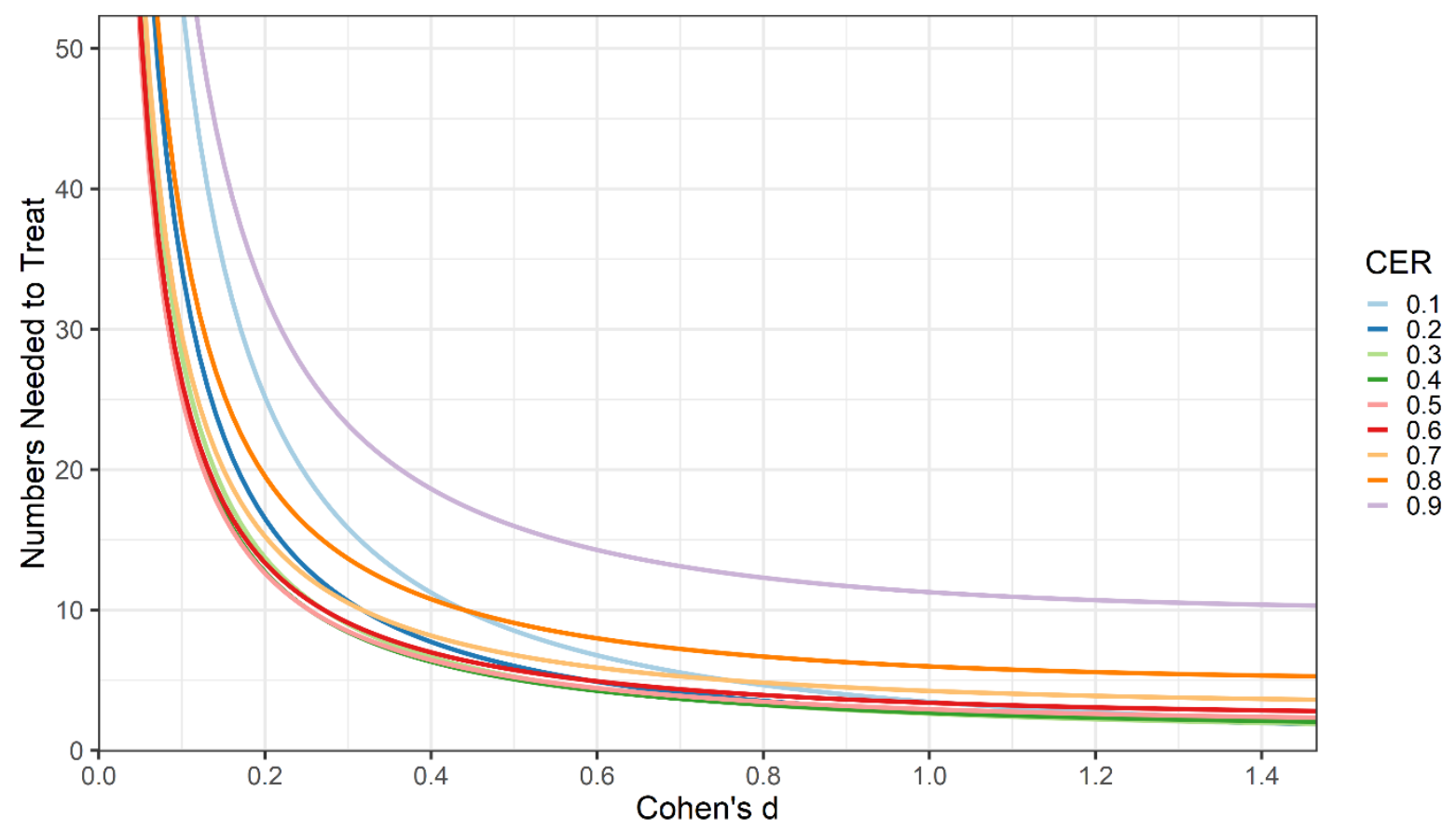

Figure 2. NNT as a function of $d$ given various control group event rates. 
It is evident from Equation 7 that researchers seeking to estimate an NNT for an intervention on a scaled outcome variable need to know two things: 1) Cohen's $d$, and 2) the CER. As discussed previously, however, the CER denotes a proportion of cases already expected to exhibit desired behavior regardless of intervention. When the variable of interest is measured dichotomously (e.g. exercising [yes/no]), a simple calculation will give the CER for the control group. However, in case of scaled outcomes (e.g. minutes spent exercising) it is less clear what proportion of the population is already exhibiting desired behavior. By creating a threshold definition, however, researchers can determine the cut-off for when behavior is considered desirable, and thus this procedure requires a dichotomization of the outcome variable to estimate the CER. In a later section, we shall discuss multiple ways to estimate the CER by applying threshold definitions. First, however, we will discuss how to obtain those threshold definitions.

\section{Obtaining threshold definitions for scaled outcomes}

Fortunately, in any context where a behavior change intervention is evaluated, there necessarily exist criteria regarding desirable behavior that warranted development of the intervention in the first place. These criteria are often established by experts exterior to the intervention developers. For example, the WHO recommendation on physical activity (World Health Organization, 2011) holds that "adults aged 18-64 should do at least 150 minutes of moderate-intensity aerobic physical activity throughout the week or do at least 75 minutes of vigorous-intensity aerobic physical activity throughout the week or an equivalent combination of moderate- and vigorous-intensity activity". The U.S. Department of Health and Human Services (U.S. Department of Health and Human Services, 2018) and the Dutch health council (Gezondheidsraad, 2017) similarly recommend a minimum of 150 minutes of moderate-intensity aerobic physical activity. 
Different countries may sometimes have different guidelines. For example, in the case of alcohol guidelines, in the Netherlands consuming 0 grams of alcohol daily is recommended (Gezondheidsraad, 2015) - and in any case at most 10 grams daily. Whereas in the United States, for women a maximum of 14 gram daily (one standard unit) and 28 grams (two standard units) for men daily is recommended (U.S. Department of Health and Human Services and U.S. Department of Agriculture, 2015). Such criteria provide concrete and tangible thresholds to define successful events in intervention research, and may often vary across specific populations.

Criteria for thresholds may also follow from consideration of policy. For instance, in our previously described example of an organizational intervention (Fleuren et al., 2018), an organization is likely to have a clearly defined participation-goal (i.e. a given percentage of minimally required participating employees), one that could serve as concrete threshold to define success. In addition to policy, practical thresholds may often may be derived from financial considerations. Some interventions may only be feasible in practice when it engenders a certain reduction of costs. For example, an intervention aimed to promote influenza vaccination amongst health-care workers (Lehmann et al, 2012, see the previous section) may be implemented to achieve a reduction of health-related absenteeism. The costreduction goal is definable by organizations, allowing a concrete estimate of the desired reduction in percentage of health-related absenteeism - in turn providing a meaningful threshold for researchers to gauge intervention effectiveness. To optimally use the NNT in applied research, a priori decisions on what outcomes are considered desired and undesired (i.e., a clear threshold) are important.

However, in some situations a realistic scenario is that there are no existing criteria allowing threshold definitions of favorable and unfavorable events in the scaled outcome. Thus, researchers may be interested in any positive change compared to the control group 
mean value. In this scenario, it is possible to estimate the NNT by reverting to the default inherent in Cohen's $d$ estimation of ES. That is, by comparing two population mean values, it estimates deviation from the control population mean that corresponds to CER $=.50$.

Therefore, if any deviation from the control group mean is taken as desirable, then $50 \%$ of the control group can be expected to show undesirable behavior and the remaining half of the population will fall in the desirable outcome range.

Notably, though, setting the threshold at the population mean value corresponding to $\mathrm{CER}=.50$ provides the most optimistic point for NNT estimation - that is, the estimated NNT will be minimal (i.e. indicative of the strongest effect) given a certain $d$ (see Figure 3), and will therefore necessarily represent an 'upper bound NNT'. In a later section, we will elaborate on the reasons for this. When CER is close to .50, the NNT will roughly approximate a frequency format of CLES. The larger the CER deviates from 0.5, the larger the discrepancy between NNT estimates and the CLES (see Table 1 for a comparison of the NNT and various probability-based effect sizes indices). That the NNT allows the CER to be variable thus represents a major advantage of NNT relative to such probability-based ES indices. Table 1 clearly illustrates this advantage of NNT over its probability-based alternatives, for example, by showing that an intervention with $d=0.2$ can nonetheless have a lower NNT than a $d=0.5$ intervention, depending on the base rate. Therefore, the optimal use of the NNT is in conjunction with a well-defined threshold definition. One that provides a meaningful cut-off point to distinguish undesirable from desirable outcomes. 
Table 1. A comparison of NNT with various probability-based effect size indices. Note that the mean in the control condition is 100 minutes of exercise; an intervention with a weak effect

\begin{tabular}{llllllll} 
d & Threshold & CER & EER & U3 & CLES & A & NNT \\
\hline 0.2 & 50 & $0.961^{\mathrm{a}}$ & $0.969^{\mathrm{b}}$ & 57.93 & 55.62 & 0.555 & 70.71 \\
\hline 0.2 & 100 & $0.489^{\mathrm{a}}$ & $0.568^{\mathrm{b}}$ & 57.93 & 55.62 & 0.555 & 12.59 \\
\hline 0.2 & 150 & $0.048^{\mathrm{a}}$ & $0.072^{\mathrm{b}}$ & 57.93 & 55.62 & 0.555 & 42.52 \\
\hline 0.5 & 50 & $0.961^{\mathrm{a}}$ & $0.984^{\mathrm{c}}$ & 69.15 & 63.82 & 0.640 & 36.81 \\
\hline 0.5 & 100 & $0.489^{\mathrm{a}}$ & $0.690^{\mathrm{c}}$ & 69.15 & 63.82 & 0.640 & 5.19 \\
\hline 0.5 & 150 & $0.048^{\mathrm{a}}$ & $0.123^{\mathrm{c}}$ & 69.15 & 63.82 & 0.640 & 13.50 \\
\hline 0.8 & 50 & $0.961^{\mathrm{a}}$ & $0.995^{\mathrm{d}}$ & 78.81 & 71.42 & 0.729 & 29.58 \\
\hline 0.8 & 100 & $0.489^{\mathrm{a}}$ & $0.814^{\mathrm{d}}$ & 78.81 & 71.42 & 0.729 & 3.44 \\
\hline 0.8 & 150 & $0.048^{\mathrm{a}}$ & $0.197^{\mathrm{d}}$ & 78.81 & 71.42 & 0.729 & 6.87 \\
\hline
\end{tabular}

Note. ${ }^{\mathrm{a}}$ Control group mean: 100 minutes of weekly exercise. ${ }^{\mathrm{b}}$ Experimental group mean: 105 minutes. ${ }^{\mathrm{c}}$ Experimental group mean: 114 minutes. ${ }^{\mathrm{d}}$ Experimental group mean: 125 minutes.

Lastly - from a science-ethics perspective and as Open Science principle - we recommend that researchers pre-register threshold decisions. This includes a pre-registration of the rationale behind the chosen threshold definition (e.g., existing evidence-based guidelines, existing policy or other practically meaningful considerations), and what cut-off point (i.e. threshold definition) on the outcome scores will be used to dichotomize the outcome in 'desired' versus 'undesired' outcomes. Such preregistration avoids post-hoc adjustments of this threshold without a sensible justification. 
The next step in calculating the NNT is to estimate the CER given a certain threshold. There are various possible strategies to obtain the threshold-corresponding CER, and we will discuss these briefly before providing concrete illustrations of the methods in the form of a tutorial.

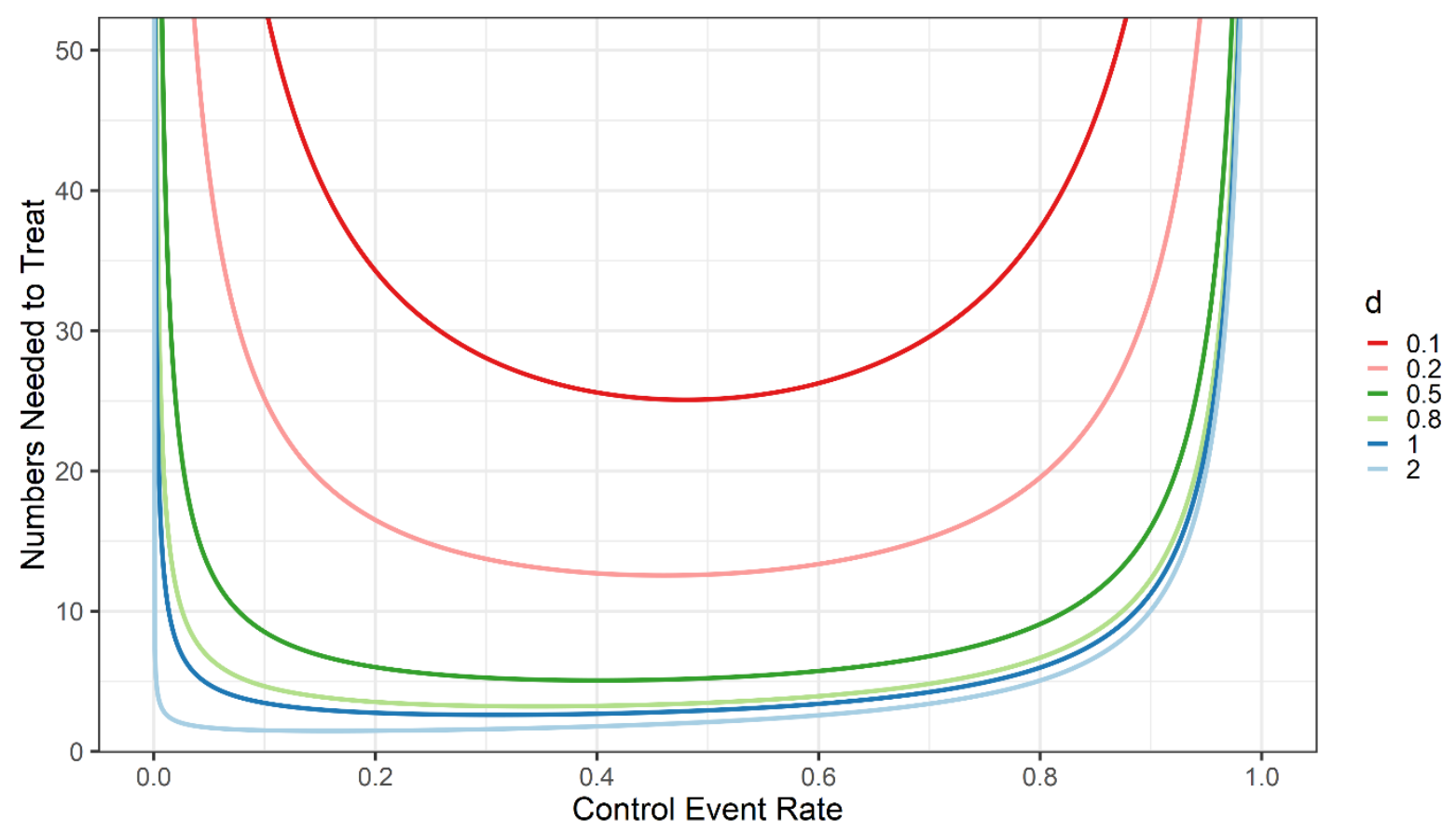

Figure 3. NNT as a function of the control event rate, given Cohen's $d$ values.

\section{Using Threshold definitions: How to estimate the CER for scaled outcomes}

When striving to estimate the NNT, one requires an estimate of the CER that corresponds with the chosen threshold for success (see Equation 7). There are at least four ways to estimate the CER once a meaningful threshold has been set: 1) using available population data, 2) using a continuous variable pre-measurement in the study, 3) using a dichotomous pre-measurement, and 4) a parametric method using summary statistics. Note that in the later tutorial examples, we shall provide concrete examples and a work-through for 
these methods. First, sometimes population data are available that provide estimates of the CER for a well-defined population (See Tutorial section, Scenario 1) so that it is not required to estimate the CER in a specific study. In the public domain there is wealth of information about specific populations and the frequency of particular behaviors. This is often the case for variables relating to public health, which are frequently monitored by institutes such as the WHO and national health institutes.

Second, a continuous baseline variable can be dichotomized using the threshold value - specifically, the proportion of cases scoring above the threshold estimates the CER (See Tutorial section, Scenario 2). This simply amounts to inspecting a frequency distribution of the control group, and observing the proportion of cases over or under a defined threshold. It may turn out, for instance, that $30 \%$ of the cases in the control group exercise for more than 150 minutes per week, which implies that a CER of .30 should be used to calculate NNT using Equation 7.

Third, one can also estimate the CER by including a dichotomous baseline measure of the outcome variable in the pre-evaluation stage of intervention research - constituting a direct binary measurement of the CER (See Tutorial section, Scenario 3). For example, assuming that we consider > 150 minutes of weekly exercise the threshold for desirable events, then one can inquire directly whether participants meet this standard in a pre-measurement of the outcome variable. This dichotomous pre-measure, in this instance, can be assessed through self-report as 'do you currently engage in 150 minutes of physical activity per week' $(0=$ no, $1=$ yes), which directly estimates $p(Y=1)$. Based on the dichotomous responses, the CER can be estimated directly for a given outcome, and can be used in Equation 7 to estimate the NNT.

Fourth, and finally, if only a mean and standard deviation are available of a data set, making an assumption about the distribution shape of the relevant variable enables computing the CER using the appropriate cumulative density function (See Tutorial, Scenario 4). For 
instance, assuming a normal distribution, the control group distribution (mean and standard deviation) can approximate the proportion of the population that reaches a threshold defining success. This is done by computing $z$-scores for the threshold values and estimating the corresponding upper-tail probability. That is,

$$
z=\frac{\text { Threshold }(C E R)-\bar{Y}}{S D(Y)}
$$

For example, assume we use a threshold defining > 150 minutes of exercise as desirable. Further assuming a mean score of 100 minutes and a standard deviation of 30 minutes, then the corresponding $z=1.667$ and the probability that a randomly selected individual will be a desirable event, i.e. $p(\mathrm{Y}=1)$, equals $p=.047$. Given sufficient $n$, then, $\mathrm{CER}=.047$. This provides an estimate of the prevalence of desired behavior without (or prior to) intervention. In short, this CER estimate is based on a normal probability function that can be restated in terms of a binomial expression; there is a $4.7 \%$ probability a randomly selected individual from the population will show the desired behavior without intervention.

\section{From information about prevalence towards effect estimation}

The CER (as estimated using the procedures discussed previously) denotes the proportion of the population that is already expected to exhibit the desired behavior, regardless of the intervention. Behavior change in the population is then a function of this CER and Cohen's $d$, that is, of the current situation and an estimate of expected change due to intervention. As can be seen in Equation 7, the size of CER is important for determining the NNT of an intervention. For a given Cohen's $d$ value, the NNT exhibits a u-shaped relationship with the CER (see Figure 4), in that behaviors that are very low in prevalence in the population (CER close to 0 ), and behaviors that are already very prevalent (CER close to 1) require a larger number of people to partake in an intervention to create behavior change of one more individual. 
Consider the physical activity example. In a population where hardly anyone exhibits the desired behavior, we require many people to partake in the intervention if we want to achieve substantive behavior change on a population level. This is also illustrated in Figure 4. The same situation applies to a population in which the majority is already conforming to researchers' intervention goals. In this sense, improving an already prevalent behavior is just as difficult as trying to improve an almost non-existent behavior. Behavior change thus requires the smallest number of participants when the CER is close to $50 \%$. This binomial thinking can be re-expressed in terms of a normal probability distribution. When a threshold corresponds to a CER of .50, this implies a large probability that a randomly picked individual exposed to the intervention will be close to (but below) the threshold defining success (in the physical activity example ' 150 minutes'). Therefore, when an intervention is implemented in a population where the threshold corresponds with a CER of 50\%, the maximal number of people can be 'pushed' over the threshold, given $d$. In Figure 4, the logic of the NNT is depicted for three different CER values. As can be seen, CER corresponds to the area under the normal curve prior to intervention. Cohen's $d$ modifies this area; the changes in event probability directly correspond to the success rate difference. Indirectly, ES measures such as RR and OR also convey information about the shift in ER from the CER towards the EER in Figure 4. The NNT can then be based on the difference in ER, and can also be computed as the inverse of the SRD. 

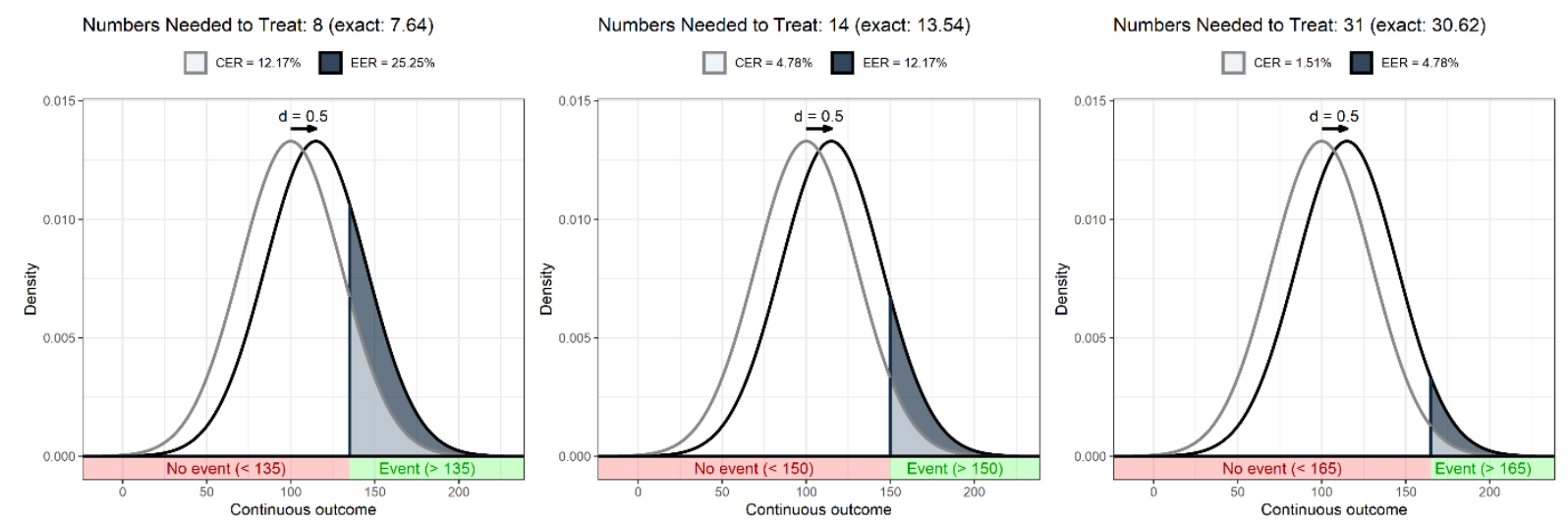

Figure 4. Estimation of NNT given a constant $d$ of 0.5. The panels illustrate why constant $d$ values can have different practical implications. The left panel approximates a CER of $12.17 \%, d$ modifies the CER towards an estimate of the EER (25.25\%). The middle panel depicts a CER of $4.78 \%$ modified towards an EER of $12.17 \%$. The right panel has a CER of $1.51 \%$ modified towards $4.78 \%$. The NNT can be estimated by dividing the total curve density (1) by the depicted change in event probability (SRD). In the left panel: SRD $=13.08$ and NNT= 8, mid panel, $\mathrm{SRD}=7.39$ and $\mathrm{NNT}=14$, and $\mathrm{SRD}=3.27$ and $\mathrm{NNT}=31$ for the right panel. The decrease in SRD and increase of NNT given a smaller CER, underscores the relevance of considering prevalence.

\section{NNT tutorial: An illustrated guide to its use}

To illustrate the procedures to estimate the NNT, we will show examples for several hypothetical situations and supplement the examples with R code that can easily be adjusted. The NNT method will be illustrated for four different scenario's. These scenario's will work through the NNT procedure using exercising behavior as the outcome variable. Parts of the data used to illustrate the method are from the actual Dutch population, other data will be simulated (and thus hypothetical) for pedagogical purposes.

\section{Scenario 1. CER population data available}

In this example we have evaluated the impact of an intervention designed to bolster exercising behavior in the general population. As discussed in a previous section, the U.S. Department of Health and Human Services (2018) recommends that adults should minimally 
exercise 2 hours and 30 minutes a week, or 30 minutes 5 days a week, consisting of moderateintensity aerobic activity. The Dutch health council (Gezondheidsraad, 2017) similarly recommends a minimum of 150 minutes of exercise per week. These values provide researchers a concrete and meaningful threshold defining desirable events (at least 150 minutes of exercise per week).

The first scenario is that there is existing population data already available - indeed, in this example, the Dutch institute of health (RIVM) has data publicly available. In the year 2017, it was estimated that roughly $47 \%$ of the general Dutch population over 4 years of age meets the threshold for desirable levels (>150 minutes) of weekly physical exercise (CBS/RIVM, 2017). In this example, therefore, the $\mathrm{CER}=.47$. Any intervention aimed at promoting physical activity in the Dutch general population could thus be tested against the backdrop of this CER. For instance, say researchers find that a specific campaign to increase minutes of exercise per week has an ES of Cohen's $d=0.5$. A simple calculation following Furukawa's equation can be done in $\mathrm{R}-$ using the 'behaviorchange' package and NNT function:

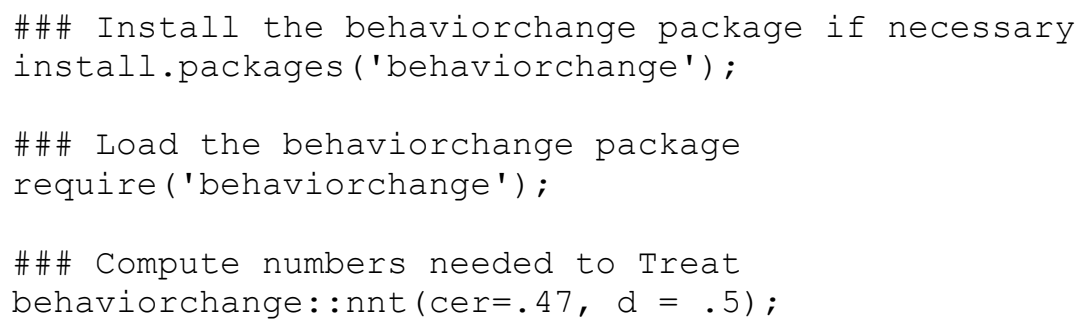

These few lines of code will results in the following values: NNT $=6$, corresponding $\mathrm{CER}=.47 \mathrm{EER}=.66$ and $\mathrm{SRD}=.19$. The NNT of 6 indicates that an intervention with an effectiveness of Cohen's $d=0.5$ could be expected to push undesirable behaviour towards desired levels of exercise in 1 out of every 6 exposed to the intervention. This can be expected to result in an overall change from $47 \%$ (CER) to $66 \%$ (EER) desirable exercise behaviour in the population. Note that the current situation regarding exercise levels in the Netherlands is from the perspective of intervention impact - close to the optimal level of CER $=.50$. When 
the population CER deviates further from .50, the impact of an intervention with given $d$ will result in larger NNT values.

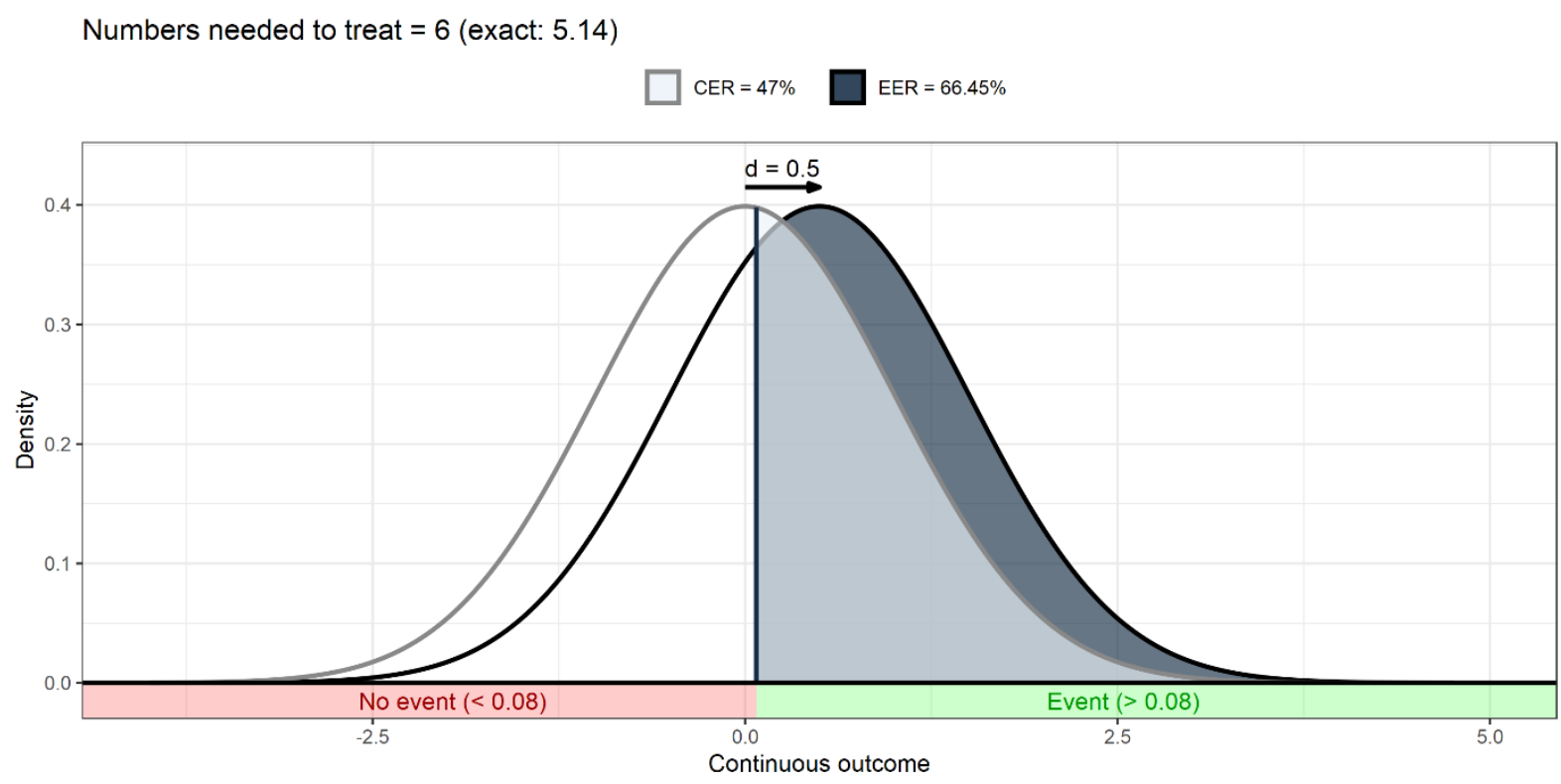

Figure 5. Results of the NNT calculation for Scenario 1.

\section{Scenario 2: Using sample data to estimate the CER}

In Scenario 1, we have used a practical threshold and corresponding CER from actual population data. It could, however, be the case that researchers managed to set a practical threshold $(>150$ minutes per week exercise) but have no access to population data on the CER. For instance, an intervention could be specifically designed to promote exercise in an above-average (say 26-30) BMI population instead of the general population. In such a case, the CER may need to be estimated in the study itself. Using simulated data, scenario 2, 3 and 4 will illustrate various methods to estimate the CER when population data are not available

A straightforward method to estimate the CER would be to inquire at baseline - or in a control condition - about minutes spent exercising. Depending on a researcher's preference (footnote), this could be assessed dichotomously (e.g. do you currently exercise $>150$ minutes per week?), or by a continuous measure (e.g. how many minutes per week do you currently engage in 
exercise?) - this variable could then be dichotomized using the preset threshold. In this scenario we assume that a continuous estimate of minutes spent exercising per week was used by the researchers. As in the primary example, the behavior data concerns exercise data, specifically, the number of minutes that above-average BMI (26-30) individuals exercise per week. Participants are considered participants with a desirable 'event' if they exercise at least 150 minutes per week. Further assume that the mean number of minutes above-average BMI participants (hypothetically) exercise is 100 , with a standard deviation of 30 . The hypothetical intervention again has an effect of Cohen's $d=0.5$ - the same ES magnitude we used in the general population intervention.

First, a dataset will be simulated, consisting of a exercising measurement collected for 1000 participants.

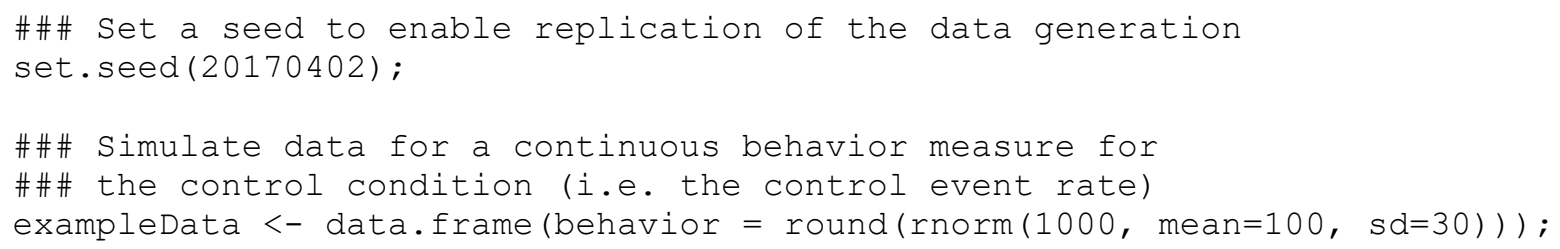

In this situation, we have a continuous behavior measurement, and we need to establish the CER. This means we first have to dichotomize this continuous measure using the threshold (= $>150$ minutes). We can then establish the event rate and use this to compute the NNT.

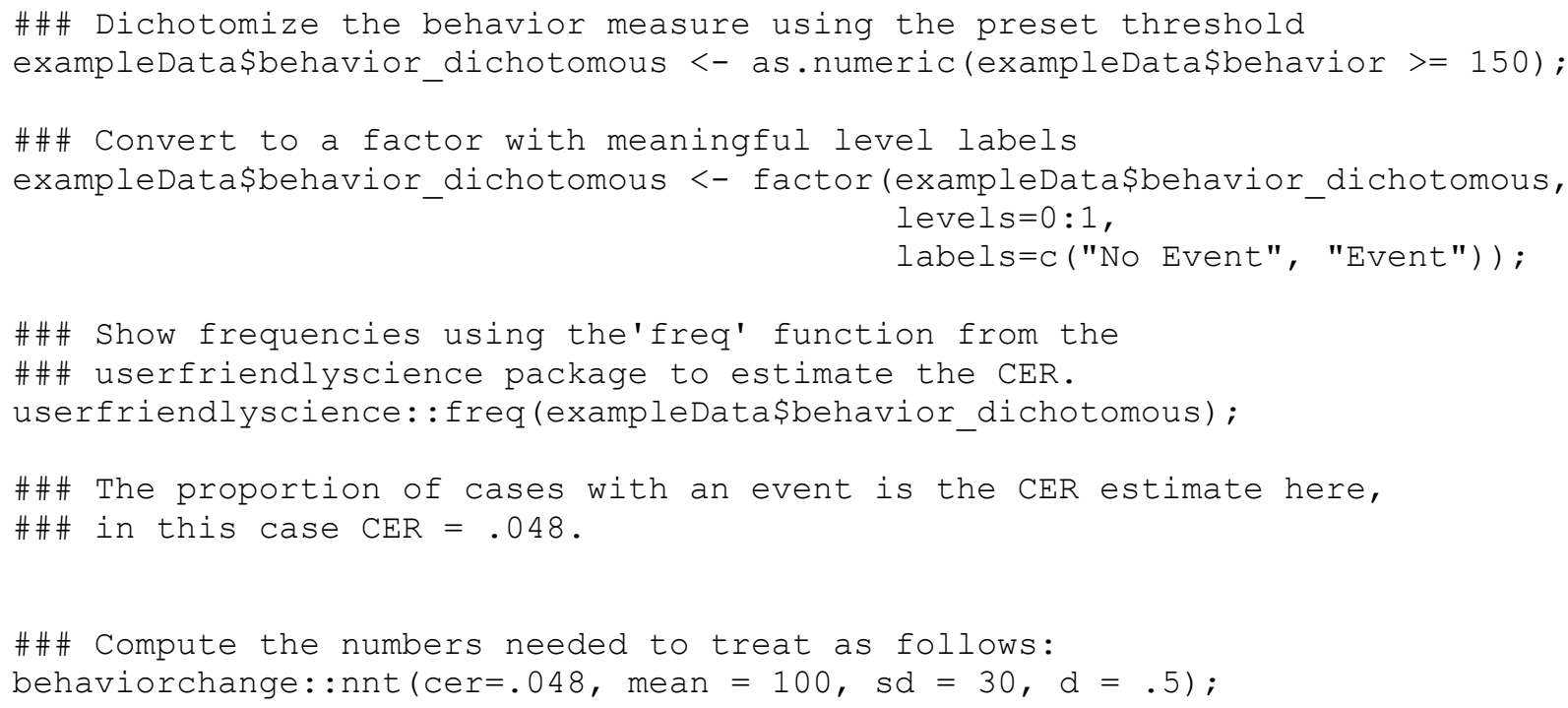


\#\#\# Note that specification of mean and sd are optional: it provides a meaningful \#\#\# $\mathrm{x}$-axis to the visualization.

In this simulated dataset, 4.8 percent of the participants in the control condition exhibited the desired behavior $(\mathrm{CER}=.048)$ - as shown in the frequency table we have generated. The result of the computation of the NNT is that 14 people must receive the intervention for one more person to change towards desired behavior $(\mathrm{NNT}=14, \mathrm{CER}=.048, \mathrm{EER}=.12, \mathrm{SRD}=.07$; see Figure 6).

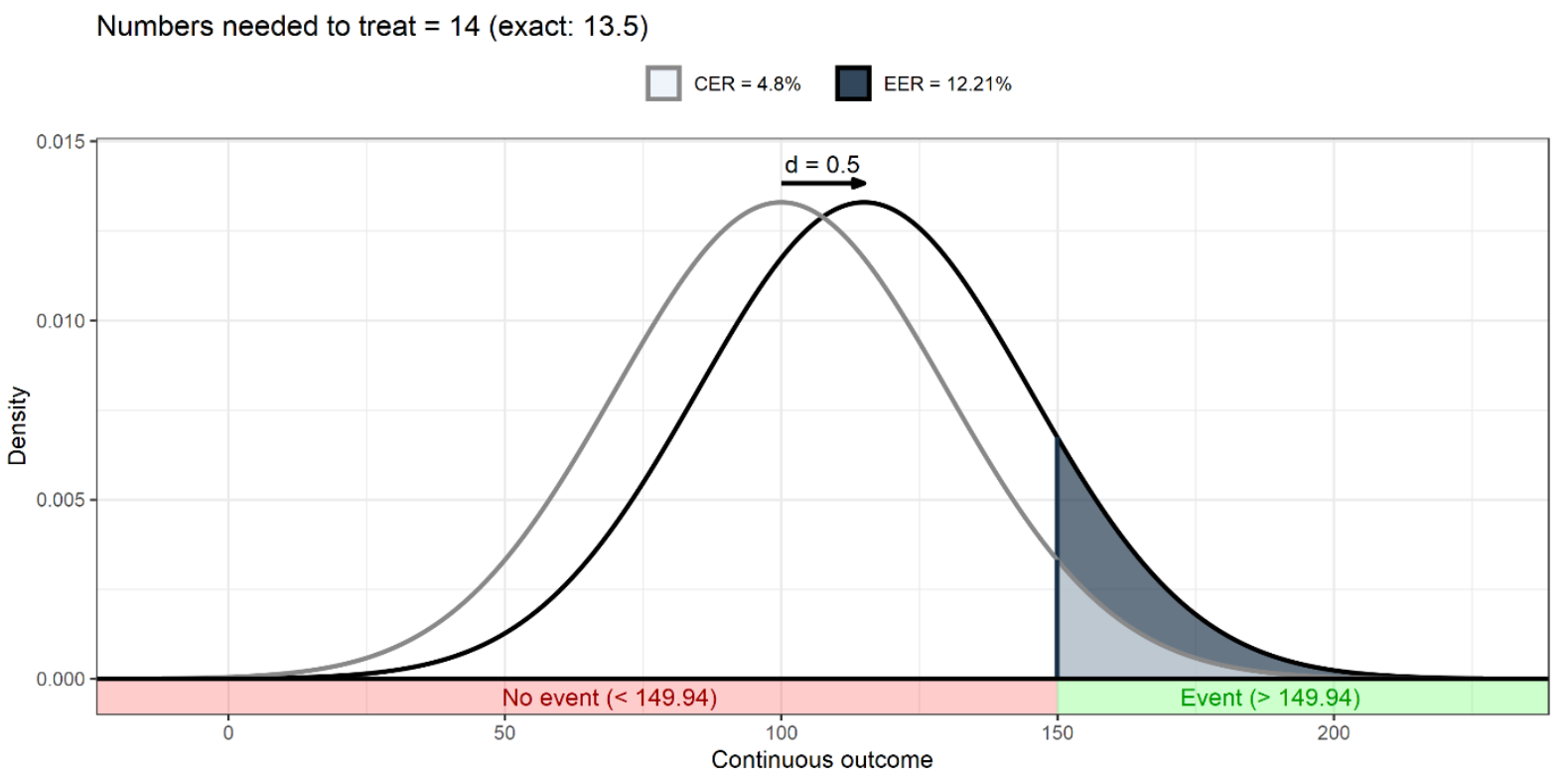

Figure 6. Results of the NNT calculation for Scenario 2.

Note the difference with our estimate of the NNT in Scenario 1. Although the intervention has the same standardized ES (Cohen's $d=.5$ ) the population impact is different for our current target population consisting of above-average BMI individuals. This is because the (hypothetical) CER has changed relative to the general population - here, fewer people are distributed around the threshold to begin with. In this population, we need to expose more people to our intervention in order to push one person above the threshold of 150 minutes exercise per week. The variable impact of an intervention with an ES of $d=.5$ on different populations highlights the added value of computing the NNT. 
As discussed in previous sections, choosing a threshold is normally based on substantive, context-specific information (e.g. minimum number of minutes one should exercise for health benefits; maximum grams of alcohol one should consume to reduce harms; maximum kilocalories to ingest in a given period). However, guidance from practice may be minimal or uncertain. Therefore, it may be useful to conduct a sensitivity analysis to gauge the effects of specifying different threshold definitions. This is possible with the thresholdSensitivity argument of the 'nnt' function:

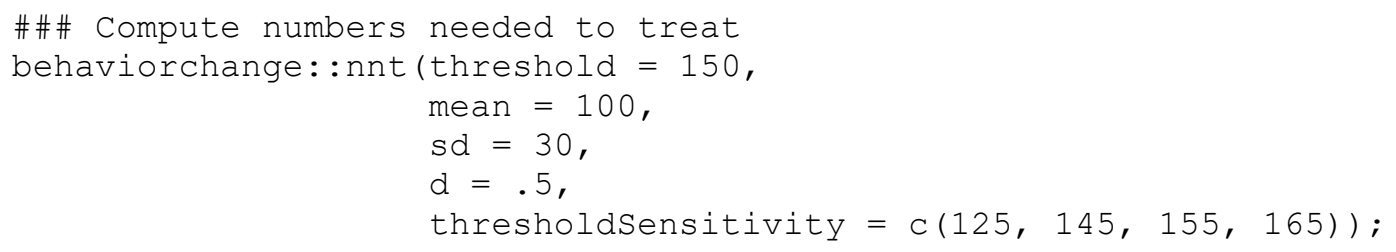

In addition to providing an estimate of the NNT and a visualization, including the 'thresholdSensitivity' function will provide a table that provides CER, EER, and NNT estimates for each specified threshold value. This allows researchers to gauge how different threshold definitions influence the NNT estimate.

\section{Scenario 3: Using a binary estimate of the CER}

Another possibility of establishing a CER (given a certain threshold definition) is to include a binary measurement in the pre-measurement / baseline condition rather than a continuous measure. For example, in the exercise example, this could be assessed by asking 'do you currently engage in more than 150 minutes of exercise per week?'. The availability of a binary variable makes computation relatively straightforward, since it is not necessary to dichotomize a variable on the threshold - rather the variable itself is assessed using the threshold definition. However, a clear downside of including a binary pre-measurement is that for other analytical purposes, continuous variables are usually preferred (e.g., Cohen, 1983; DeCoster et al., 2009). The binary variable we created in Scenario 2 is a variable that would be available to researchers including a binary CER measurement in a study. Were such a 
variable available, it is possible to directly request frequencies and inspect the proportion of cases in the yes-category to estimate CER. Accordingly, this value can be entered in the NNT equation by first estimating the proportion.

\#\#\# Show the frequencies

userfriendlyscience: : freq (exampleData\$behavior_dichotomous) ;

\#\#\# Compute numbers needed to treat

behaviorchange: : nnt (cer $=.048$, mean $=100, \mathrm{sd}=30, \mathrm{~d}=.5)$;

The results are identical, of course, to those in Scenario 2 (see Figure 6).

Scenario 4: Using summary data (if no raw data are available)

This is a different example: here, a researcher has no primary data about the control event rate. Instead, only information about the distribution is available: a mean and a standard deviation. This scenario occurs, for example, when a researcher wants to estimate the NNT for a study conducted by others. Making appropriate distributional assumptions (e.g. assuming a normal distribution) then enables estimating the percentage of the distribution that exceeds the threshold.

We will again use the above-average BMI data, assuming a mean of 100 minutes, a standard deviation of 30 minutes, a threshold of 150 minutes per week, and an ES estimate of Cohen's $d=0.5$ for our intervention to promote exercising. When assuming normally distributed data, however, these numbers can just be directly provided to the NNT function.

\#\#\# Compute numbers needed to treat

behaviorchange: : nnt (threshold=150, mean $=100, \mathrm{sd}=30, \mathrm{~d}=.5$ );

In this situation, the CER is computed at $4.78 \%$, and the EER at $12.17 \%$, resulting in an estimated NNT=14 (see Figure 7). 


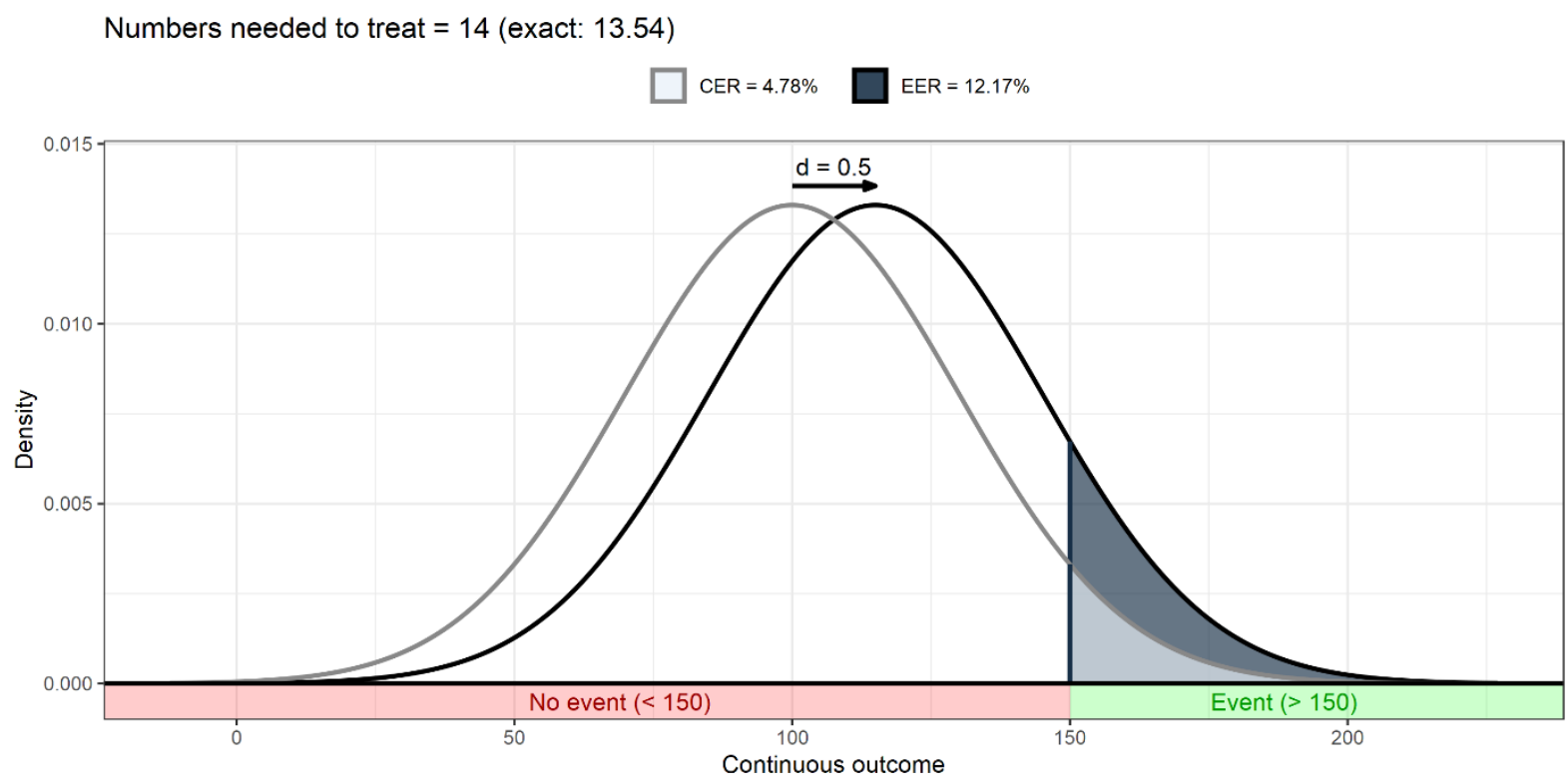

Figure 7. Results of the NNT calculation for Scenario 4.

\section{Discussion}

We have described the NNT as an additional index of ES for behavioral interventionists, one that conveys practical and intuitive information about intervention effectiveness. The NNT is a step beyond the relative ES measures, such as Cohen's $d$, by which interventions are commonly evaluated. The present paper has argued for two benefits of NNT estimation for applied research: 1) it has the potential to inform policy and practice about the benefits of implementing an intervention because it expresses ES in a commonsense frequency-format (number of people), and 2) the measure considers prevalence of behavior prior to intervention, thereby relating it more directly to intervention impact on a specific population. In short, the NNT brings intervention effectiveness analysis closer to the real-world implications of interventions.

Reshaping common indices of effect in measures that use the NNT metric may also lead to different interpretations of ES. In psychological science, the coefficient of determination in regression models has a realistic range of $0-.40$. Relatedly, in the analysis of variance context, variance-based indices such as eta-squared and omega-squared seldom 
approach this level of explained variance. In practice, such measures therefore make it almost impossible to meaningfully differentiate between the effectiveness of intervention. Rosenthal \& Rubin, 1982) went so far so as to argue that $r$ or $r^{2}$ - based categories of 'small effect' (e.g. $r=.30$ ) can lead to "absurd" conclusions about effect magnitude. In the BESD, a correlation of .30 corresponds to estimated success rate difference of $30 \%$. Reducing the probability of 'death' with $30 \%$, these authors argued, can hardly be seen a small feat. Given that BESD frames ES in optimal base-rate circumstances, however, the conclusion may be too strong (see McGraw, 1991). Nonetheless, the BESD illustrates that the effectiveness of behavioral interventions may be consistently - and incorrectly - be interpreted as 'small' given traditional indices. Furukawa also notes that Cohen's categorization of ES may lead to inadequate conclusions when authors argue at the population level. Using the frequency of antidepressant treatment in Japan (about 2 million) and given NNT=17 the author points out: 'If we can find a new treatment that is better than the current treatment as usual by Cohen's $d$ of 0.2 , it can bring about remission in additional 100 thousand or more people who would not have done so on the current treatment. This of course is no trivial number' (p.5).

The arguments we have provided to supplementing statistical analysis with the NNT are focused on research with an applied orientation, clearly applicable to health intervention research but generalizable to other disciplines interested in real-world implications of intervention. Notably, the arguments to go beyond NHST and traditional ES indices are framed as psychological rather than statistical; it allows a better judgement of population impact. By doing so, practical considerations such as cost-effectiveness are put within grasp (see Sinclair, Cook, Guyatt, Pauker, \& Cook, 2001), because 'number of people' can be readily transformed to an estimation of costs ( $c f$. standardized change in means). Hence, the NNT can be seen as "the poor man's cost-effectiveness estimate": in situations where resources to conduct a cost-effectiveness study lack, the NNT is often within reach. 
Our description of the NNT estimate based on the NNT index has several implications for research practice. First, Equation 7 (FL) emphasizes and illustrates the importance of the CER in determining the NNT. Informally, such base-rates of behavior are often implicitly considered in defining target groups, in which a problematic behavior has sufficient incidence to warrant intervention (and therefore, a higher CER). A formalization of the intuition that prevalence of desired versus undesired behavior is a modifier of effectiveness is provided by the NNT. It enables a better prediction of the ES that is needed for behavior change with impact in the real-world. The tutorial (Scenario 2 and 3) illustrates how an intervention with a given effectiveness may differentially impact specific target populations. To take intervention analysis out of abstract quantifications into information with practical value, researchers need to incorporate well-defined threshold definitions of intervention success in intervention research, and a measure of how many people already meet this criterion.

\section{Some limitations of the procedure}

The NNT as presented here works under the assumptions of normality and homogeneity of variances. The procedure further involves a categorization of quantitative data. From a statistical optimization perspective, categorization of quantitative data is always a suboptimal method of data processing, since information is lost in the process (Cohen, 1983; DeCoster et al., 2009; MacCallum et al., 2002). Dichotomizing a scaled outcome variable tends to inflate error variance, in turn resulting in attenuation of the estimated effect size. Therefore, in cases where the outcome variable is substantially affected by measurement error, it is likely that the sample NNT estimate will be an underestimate of the true NNT. For interventions using observed variables (such as self-reported exercise behavior) as outcome, this may be less problematic compared to studies interested on intervention effects on latent variables (usually, psychological variables). In cases where latent variables are involved, it is warranted to adjust the estimated Cohen's $d$ for measurement error (for a method see Bobko, 
Roth, \& Bobko, 2001) - before using it in Equation 7 to obtain the NNT. This prevents measurement error from leading to overly conservative NNT estimates.

As Kraemer \& Kupfer (2006) put the dilemma: 'The choice is often depicted as a struggle between statistical considerations favoring dimensional outcomes and clinical considerations favoring categorical outcomes.' (p.994). However, we propose that for several reasons a focus on practical significance, as expressed by the NNT, does in fact not generate any such decision-making dilemma. First, optimality in statistical methods faces a trade-off with communicative and practical utility of their metrics - particularly so for applied research. In our view, the benefits of the NNT in intuitively conveying information about intervention effects outweigh the loss of information inherent to the procedure. Second, standardized mean change measures (e.g. Cohen's $d$ ), may from a practical perspective be regarded as similar categorical indices of ES. Researchers do not draw and report implications and interpretations using dimensional indices, but rather categorize them into informational packages of 'small', 'medium' and 'large'. The merit of NNT, then, should best be compared to traditional indices on the level of rule-of-thumbs for size interpretation. Evaluated as such, the NNT is a clear valuable addition to current practice.

Since the value of the NNT lies on a different dimension than statistical considerations, we suggest that the measure should not be contrasted with, or serve as alternative, to common measures of ES. Instead, we recommend that researchers use the NNT measure as an additional (practical) dimension of intervention analysis, to be used in conjunction with indices such as Cohen's $d$. In line with previous recommendations (e.g., Baguley, 2009; Wilkinson et al., 1999), then, we suggest that applied researchers communicate ES magnitude on three levels: using a) unstandardized and b) standardized units, but also c) incorporate the NNT to express population impact. By extension of these recommendations, we do not promote replacement of scaled variables with binary outcome 
measures with the aim of increasing ease of NNT calculation. Instead, we suggest that moving beyond NHST implies more than a shift towards traditional ES indices that express the relevance of an effect in terms of abstract quantities - especially for those studies that are of an applied nature. Additional reporting of NNT may be one tool to narrow the gap between applied psychological research and practice. 


\section{References}

Altman, D. G. (1998). Confidence intervals for the number needed to treat. Bmj, 317(7168), 1309-1312. http://doi.org/10.1136/bmj.317.7168.1309

Altman, D. G., \& Andersen, P. K. (1999). Calculating the number needed to treat for trials where the outcome is time to an event. BMJ, 319(7223), 1492-1495. http://doi.org/10.1136/bmj.319.7223.1492

APA. (2015). Advice for translating science into policy. Vol 46, No. 4, 11. Retrieved from https://www.apa.org/monitor/2015/04/upfront-advice.aspx

Austin, P. C. (2010). Absolute risk reductions, relative risks, relative risk reductions, and numbers needed to treat can be obtained from a logistic regression model. Journal of Clinical Epidemiology, 63(1), 2-6. http://doi.org/10.1016/j.jclinepi.2008.11.004

Baguley, T. (2004). Understanding statistical power in the context of applied research. Applied Ergonomics, 35(2), 73-80. http://doi.org/10.1016/j.apergo.2004.01.002

Baguley, T. (2009). Standardized or simple effect size: What should be reported? British Journal of Psychology, 100(3), 603-617. http://doi.org/10.1348/000712608X377117

Bartholomew Eldregde, L. K., Markham, C. M., Ruiter, R. A. C., Fernàndez, M. E., Kok, G., Parcel, G. S., ... Parcel, G. S. (2016). Planning health promotion programs: An Intervention Mapping approach (4th ed.). Hoboken, NJ: Wiley.

Bobko, P., Roth, P. L., \& Bobko, C. (2001). Correcting the Effect Size of d for Range Restriction and Unreliability. Organizational Research Methods, 4(1), 46-61. http://doi.org/10.1177/109442810141003

Brooks, M. E., Dalal, D. K., \& Nolan, K. P. (2014). Are common language effect sizes easier to understand than traditional effect sizes? Journal of Applied Psychology, 99(2), 332340. http://doi.org/10.1037/a0034745

CBS/RIVM. (2017). Leefstijl monitor. Retrieved from https://www.volksgezondheidenzorg.info/onderwerp/sport-en-bewegen/cijferscontext/huidige-situatie\#node-beweegrichtlijnen

Citrome, L., \& Ketter, T. A. (2013). When does a difference make a difference? Interpretation of number needed to treat, number needed to harm, and likelihood to be helped or 
harmed. International Journal of Clinical Practice. http://doi.org/10.1111/ijcp.12142

Cohen, J. (1983). The Cost of Dichotomization. Applied Psychological Measurement, 7(3), 249-253. http://doi.org/10.1177/014662168300700301

Cohen, J. (1988). Statistical power for the social sciences. Hillsdale, NJ: Laurence Erlbaum and Associates.

Cohen, J. (1994). The earth is round ( $\mathrm{p}$ <.05). American Psychologist, 49(12), 997-1003. http://doi.org/10.1037/0003-066X.49.12.997

Cook, R. J., \& Sackett, D. L. (1995). The Number Needed to Treat - a Clinically Useful Measure of Treatment Effect. British Medical Journal, 310(6977), 452-454.

Cosmides, L., \& Tooby, J. (1996). Are humans good intuitive statisticians after all? Rethinking some conclusions from the literature on judgment under uncertainty. Cognition, 58(1), 1-73. http://doi.org/10.1016/0010-0277(95)00664-8

Cumming, G. (2014). The new statistics: Why and how. Psychological Science, 25(1), 7-29. http://doi.org/10.1177/0956797613504966

Da Costa, B. R., Rutjes, A. W. S., Johnston, B. C., Reichenbach, S., Nuesch, E., Tonia, T., ... Juni, P. (2012). Methods to convert continuous outcomes into odds ratios of treatment response and numbers needed to treat: Meta-epidemiological study. International Journal of Epidemiology, 41(5), 1445-1459. http://doi.org/10.1093/ije/dys124

DeCoster, J., Iselin, A.-M. R., \& Gallucci, M. (2009). A conceptual and empirical examination of justifications for dichotomization. Psychological Methods, 14(4), 349366. http://doi.org/10.1037/a0016956

Del re, A. C. (2013). compute.es: Compute effect Sizes. Retrieved from http://cran.rproject.org/web/packages/compute.es

Fleuren, B. P. I., De grip, A., Kant, I., \& Zijlstra, F. (2018). Time equals money? A randomized controlled field experiment on the effects of four types of vouchers on training participation. Personal Communication.

Furukawa, T. A. (1999). From effect size into number needed to treat. Lancet, 353(9165), 1680. http://doi.org/10.1016/s0140-6736(99)01163-0le 
Furukawa, T. A., \& Leucht, S. (2011). How to Obtain NNT from Cohen's d: Comparison of two methods. PLoS ONE, 6(4), 2-6. http://doi.org/10.1371/journal.pone.0019070

Gardner, M. J., \& Altman, D. G. (1986). Statistics in Medicine Confidence intervals rather than P values: estimation rather than hypothesis testing. British Medical Journal (Clinical Research Ed.), 292(6522), 746-750. http://doi.org/10.1136/bmj.292.6522.746

Gezondheidsraad. (2015). Richtlijnen goede voeding. Retrieved from https://www.gezondheidsraad.nl/documenten/adviezen/2015/11/04/richtlijnen-goedevoeding-2015

Gezondheidsraad. (2017). Beweegrichtlijnen 2017. Den Haag. Retrieved from https://www.gezondheidsraad.nl/documenten/adviezen/2017/08/22/beweegrichtlijnen2017

Gigerenzer, G. (1996). Why do frequency formats improve Bayesian reasoning? Cognitive algorithms work on information, which needs representation. Behavioral and Brain Sciences, 19(1), 23. http://doi.org/10.1017/S0140525X00041248

Gigerenzer, G., Hoffrage, U., Mellers, B. A., \& McGraw, A. P. (1995). How to Improve Bayesian Reasoning Without Instruction: Frequency Formats. Psychological Review, 102(4), 684-704. http://doi.org/10.1037/0033-295X.102.4.684

Greenland, S., Senn, S. J., Rothman, K. J., Carlin, J. B., Poole, C., Goodman, S. N., \& Altman, D. G. (2016). Statistical tests, $P$ values, confidence intervals, and power: a guide to misinterpretations. European Journal of Epidemiology, 31(4), 337-350. http://doi.org/10.1007/s10654-016-0149-3

Gruijters, S. L. K. (2016). Baseline comparisons and covariate fishing: Bad statistical habits we should have broken yesterday. European Health Psychologist, 18(5), 205-209.

Hasselblad, V., \& Hedges, L. V. (1995). Meta-analysis of screening and diagnostic tests. Psychological Bulletin, 117(1), 167-178. http://doi.org/10.1037/0033-2909.117.1.167

Hilton, D. J., Reid, C. M., \& Paratz, J. (2006). An under-used yet easily understood statistic: the number needed to treat (NNT). Physiotherapy, 92(4), 240-246. http://doi.org/10.1016/j.physio.2006.06.003

Hsu, L. M. (2004). Biases of Success Rate Differences Shown in Binomial Effect Size 
Displays. Psychological Methods, 9(2), 183-197. http://doi.org/10.1037/1082989X.9.2.183

Kahneman, D., Slovic, P., \& Tversky, A. (1974). Judgment under uncertainty: heuristics and biases. Science, 185(4157), 1124-1131. http://doi.org/10.1126/science.185.4157.1124

Kahneman, D., \& Tversky, A. (1972). Subjective Probability: A Judgement of Representativeness. Cognitive Psychology, 3, 430-454. http://doi.org/10.1016/00100285(72)90016-3

Kirk, R. E. (1996). Practical Significance: A Concept Whose Time Has Come. Educational and Psychological Measurement, 56(5), 746-759. http://doi.org/10.1177/0013164496056005002

Kraemer, H. C., \& Kupfer, D. J. (2006). Size of treatment effects and their importance to clinical research and practice. Biological Psychiatry, 59(11), 990-996. http://doi.org/S0006-3223(05)01210-2 [pii]

Lakens, D. (2013). Calculating and reporting effect sizes to facilitate cumulative science: a practical primer for t-tests and ANOVAs. Frontiers in Psychology, 4. http://doi.org/10.3389/fpsyg.2013.00863

Laupacis, A., Sackett, D. L., \& Roberts, R. S. (1988). An Assessment of Clinically Useful Measures of the Consequences of Treatment. New England Journal of Medicine, 318(26), 1728-1733. http://doi.org/10.1056/NEJM198806303182605

Lehmann, B. A., Chapman, G. B., Franssen, F. M. E., Kok, G., \& Ruiter, R. A. C. (2016). Changing the default to promote influenza vaccination among health care workers. Vaccine, 34(11), 1389-1392. http://doi.org/10.1016/j.vaccine.2016.01.046

MacCallum, R. C., Zhang, S., Preacher, K. J., \& Rucker, D. D. (2002). On the practice of dichotomization of quantitative variables. Psychological Methods, 7(1), 19-40. http://doi.org/10.1037//1082-989X.7.1.19

McGraw, K. O. (1991). Problems with the BESD: A comment on Rosenthal's "How are we doing in soft psychology?” American Psychologist, 46(10), 1084-1086. http://doi.org/10.1037/0003-066X.46.10.1084

McGraw, K. O., \& Wong, S. P. (1992). A common language effect size statistic. 
Psychological Bulletin, 111(2), 361-365. http://doi.org/10.1037/0033-2909.111.2.361

Niiniluoto, I. (1993). The aim and structure of applied research. Erkenntnis, 38(1), 1-21. http://doi.org/10.1007/BF01129020

Pek, J., \& Flora, D. B. (2018). Reporting effect sizes in original psychological research: A discussion and tutorial. Psychological Methods, 23(2), 208-225. http://doi.org/10.1037/met0000126

Peters, G.-J. Y. (2019). Behaviorchange: tools for behavior change researchers and professionals. Retrieved from https://a-bc.eu/R/behaviorchange

Peters, G.-J. Y., \& Crutzen, R. (2017). Knowing exactly how effective an intervention, treatment, or manipulation is and ensuring that a study replicates: accuracy in parameter estimation as a partial solution to the replication crisis. http://doi.org/10.31234/osf.io/cjsk2

R Development Core Team. (2017). R: A Language and Environment for Statistical Computing. Vienna, Austria.

Rosenthal, R. (1990). How are we doing in soft psychology? American Psychologist, 45, 775777. http://doi.org/10.1037/0003-066X.45.6.775

Rosenthal, R. (1991). Effect sizes: Pearson's correlation, its display via the BESD, and alternative indices. American Psychologist, 46(10), 1086-1087. http://doi.org/10.1037//0003-066X.46.10.1086

Rosenthal, R. (2005). Binomial Effect Size Display. In Encyclopedia of Statistics in Behavioral Science. Chichester, UK: John Wiley \& Sons, Ltd. http://doi.org/10.1002/0470013192.bsa050

Rosenthal, R., \& Rubin, D. D. B. (1982). A simple, general purpose display of magnitude of experimental effect. Journal of Educational Psychology, 74(2), 166-169. Retrieved from http://doi.apa.org/journals/edu/74/2/166.pdf

Ruscio, J. (2008). A Probability-Based Measure of Effect Size: Robustness to Base Rates and Other Factors. Psychological Methods, 13(1), 19-30. http://doi.org/10.1037/1082989X.13.1.19 
Ruscio, J., \& Gera, B. L. (2013). Generalizations and Extensions of the Probability of Superiority Effect Size Estimator. Multivariate Behavioral Research, 48(2), 208-219. http://doi.org/10.1080/00273171.2012.738184

Ruscio, J., \& Mullen, T. (2012). Confidence Intervals for the Probability of Superiority Effect Size Measure and the Area Under a Receiver Operating Characteristic Curve. Multivariate Behavioral Research, 47(2), 201-223. http://doi.org/10.1080/00273171.2012.658329

Sinclair, J. C., Cook, R. J., Guyatt, G. H., Pauker, S. G., \& Cook, D. J. (2001). When should an effective treatment be used? Journal of Clinical Epidemiology, 54(3), 253-262. http://doi.org/10.1016/S0895-4356(01)00347-X

Sloman, S. A., Over, D., Slovak, L., \& Stibel, J. M. (2003). Frequency illusions and other fallacies. Organizational Behavior and Human Decision Processes, 91(2), 296-309. http://doi.org/10.1016/S0749-5978(03)00021-9

Suissa, S. (1991). Binary methods for continuous outcomes: A parametric alternative. Journal of Clinical Epidemiology, 44(3), 241-248. http://doi.org/10.1016/0895-4356(91)90035-8

Thorlund, K., Walter, S. D., Johnston, B. C., Furukawa, T. a., \& Guyatt, G. H. (2011). Pooling health-related quality of life outcomes in meta-analysis-a tutorial and review of methods for enhancing interpretability. Research Synthesis Methods, 2(3), 188-203. http://doi.org/10.1002/jrsm.46

Torchiano, M. (2017). effsize: Efficient Effect Size Computation. Retrieved from https://cran.r-project.org/package=effsize

Tversky, A., \& Kahneman, D. (1983). Extensional vs. intuitive reasoning: The conjunction falllacy in probability judgment. Psychological Review, 90, 293-315.

U.S. Department of Health and Human Services. (2018). Physical Activity Guidelines for Americans Title. Washinton, D.C, U.S. Retrieved from https://health.gov/paguidelines/secondedition/pdf/Physical_Activity_Guidelines_2nd_edition.pdf

U.S. Department of Health and Human Services and U.S. Department of Agriculture. (2015). Dietary Guidelines for Americans (8th edition). Retrieved from 
https://health.gov/dietaryguidelines/2015/guidelines/.

Vargha, A., \& Delaney, H. D. (2000). A Critique and Improvement of the CL Common Language Effect Size Statistics of McGraw and Wong. Journal of Educational and Behavioral Statistics, 25(2), 101-132. http://doi.org/10.3102/10769986025002101

Wilkenson and the taskforce on statistical inference. (1999). Statistical methods in psychology journals: Guidelines and explanations. American Psychologist, 54(8), 594-604.

Wilkinson, L., Aiken, L., Appelbaum, M., Boodoo, G., Kenny, D. A., Kraemer, H., ... Cronbach, L. (1999). Statistical methods in psychology journals: Guidelines and explanations. American Psychologist, 54(8), 594-604. http://doi.org/10.1037/0003066X.54.8.594

World Health Organization. (2011). Global Recommendation on Physical Activity for Health. Retrieved December 16, 2018, from https://www.who.int/dietphysicalactivity/physicalactivity-recommendations-18-64years.pdf 
Figure captions.

Figure 1. Basic binary effect size computations and relations.

SRD, AUC and NNT conversions are based on Kraemer \& Kupfer (2006). Abbreviations: OR $=$ Odds Ratio; RR = Relative Risk; SRD = Success Rate Difference; ARR= Absolute Risk Reduction; $\mathrm{EER}=$ Experimental Event Rate; $\mathrm{CER}=$ Control Event Rate; $\mathrm{AUC}=$ Area Under the $($ ROC) Curve; NNT = Numbers Needed to Treat.

Figure 2. NNT as a function of $d$ given various control group event rates.

Figure 3. NNT as a function of the control event rate, given Cohen's $d$ values.

Figure 4. Estimation of NNT given a constant $d$ of 0.5 . The panels illustrate why constant $d$ values can have different practical implications. The left panel approximates a CER of $20 \%, d$ modifies the CER towards an estimate of the EER (37\%). The middle panel depicts a CER of $50 \%$ modified towards an EER of $69 \%$. The right panel has a CER of $80 \%$ modified towards $91 \%$. The NNT can be estimated by dividing the total curve density (1) by the depicted change in event probability (SRD). In Panel A: SRD $=0.17$ and NNT $=7$, Panel B, SRD = 0.19 and $\mathrm{NNT}=5$, and for Panel $\mathrm{C}$ the $\mathrm{SRD}=0.11$ and $\mathrm{NNT}=9$.

Figure 5. Results of the NNT calculation for Scenario 1.

Figure 6. Results of the NNT calculation for Scenario 2.

Figure 7. Results of the NNT calculation for Scenario 4. 